\title{
NILPOTENT SPACES: SOME INEQUALITIES ON NILPOTENCY DEGREES
}

\author{
AUGUSTO REYNOL FILHO
}

(Communicated by Frederick R. Cohen)

\begin{abstract}
Our purpose in this work is to compare the nilpotency degree of the action of $\pi_{1}(X)$ on $\pi_{n}(X) \quad(2 \leq n \leq 7)$ with the one of the action of $\pi_{1}(X)$ on $\operatorname{Hn}(\widetilde{X})$. We work in the category of the nilpotent spaces (here $\tilde{X}$ means the universal cover of $X$ ). The main point in the proof of the main theorems, which yields such inequalities, is the reiterated use of the Serre spectral sequence.
\end{abstract}

\section{INTRODUCTION}

The idea of dealing with the subject considered in this paper arose in a talk between the author and Professor Peter Hilton.

In [HMR] the authors proved that if $X$ is a connected CW-complex and $\pi_{1}(X)$ is nilpotent, then $\pi_{1}(X)$ acts nilpotently on $\pi_{n}(X) \quad(n \geq 2)$ if and only if $\pi_{1}(X)$ acts nilpotently on $H_{n}(\widetilde{X}) \quad(n \geq 2)$ where $\widetilde{X}$ is the universal cover of $X$.

This fact gave rise to a natural question: compare the nilpotency degrees of the actions mentioned in the theorem above. It was already known to the authors that $\pi_{1}(X)$ acted on $\pi_{2}(X)$ and on $H_{2}(\widetilde{X})$ with the same nilpotency degree, due to Hurewicz's Theorem that $\pi_{2}(X) \cong H_{2}(\widetilde{X})$.

It remained, therefore, to investigate the case $n \geq 3$. This is exactly the problem that Professor Hilton suggested we study.

We have been successful in finding inequalities, when $n \leq 7$, between nil $_{\pi_{1}(X)} \pi_{n}(X)$ (the nilpotency degree of the action of $\pi_{1}(X)$ on $\pi_{n}(X)$ ) and nil $_{\pi_{1}(X)} H_{n}(\widetilde{X})$, and this is the subject of this paper. The inequalities we obtained are the content of Theorem 12 , which is our main result.

This work is part of the author's Ph.D. thesis done under the guidance of Professor Peter John Hilton.

The author is very much indebted to Professor Daciberg Lima Gonçalves, at whose suggestion this work was developed.

Let $\pi \stackrel{w}{\longrightarrow} \operatorname{Aut}(A)$ be an action of a group $\pi$ on an abelian group $A$.

We recall that $w$ yields, $\forall n \geq 0$, an action $w_{n}$ of $\pi$ on $H_{n}(K(A, m)$ ) (here $m$ is a fixed integer, $m \geq 1$, and $K(A, m)$ is an Eilenberg-MacLane space),

Received by the editors October 18, 1989.

1991 Mathematics Subject Classification. Primary 55P60. 
defined by

$(\forall x \in \pi) \exists !\left[f_{x}\right] \in[K(A, m), * ; K(A, m), *]$ such that $f_{x^{*}}=w(x)$.

(For a more detailed description see [W, pp. 100, 225]).

We define $w_{n}(x)=f_{x^{*}}: H_{n}(K(A, m)) \rightarrow H_{n}(K(A, m))$.

The proof of the first proposition is known (see [HMR, Lemma II.2.17]). We decided, nevertheless, to present it here owing to the technique used in its proof, which is going to be used repeatedly in the sequel.

Proposition 1. $w$ nilpotent $\Rightarrow w_{n}$ nilpotent, $\forall n \geq 0$.

Proof. We argue by induction on $c=$ nil $w=\operatorname{nil}_{\pi} A$.

If $c=1$ it follows from the definition that $w_{n}$ is trivial and therefore nilpotent.

If $c>1$ we take $\Gamma=\Gamma_{w}^{c} \neq(0)$ (here we follow the convention of [HMR]), and the fibration

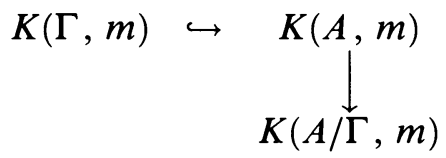

This fibration gives rise to a spectral sequence (Serre) in which $E_{r, s}^{2}=$ $H_{r}\left(K(A / \Gamma, m) ; H_{s}(K(\Gamma, m))\right)$. (Here, we should point out that the homology is taken with trivial coefficients, since if $m \geq 2$, the base is simply connected and if $m=1$ we obtain $H_{r}\left(A / \Gamma ; H_{s}(\Gamma)\right)$ and $A / \Gamma$ acts trivially on $\Gamma$, so that $A / \Gamma$ acts trivially on $\left.H_{s}(\Gamma).\right)$

We now invoke the Universal Coefficient Theorem to get the exact sequence

$$
\begin{aligned}
& H_{r}(K(A / \Gamma, m)) \otimes H_{s}(K(\Gamma, m)) \\
& \quad \longrightarrow E_{r, s}^{2} \rightarrow \operatorname{Tor}\left(H_{r-1}(K(A / \Gamma, m)), H_{s}(K(\Gamma, m))\right) .
\end{aligned}
$$

Taking into account the induction hypothesis, Lemma 1.1 from $[\mathrm{H}]$ and Proposition I.4.3 on p. 35 of [HMR], we can claim that $\pi$ acts nilpotently on $E_{r, s}^{2}$.

Repeated use of Proposition I.4.3 on p. 35 of [HMR] assures that $\pi$ acts nilpotently on $E_{r, s}^{\infty}$, and therefore on $H_{n}(K(A, m))$.

Lemma 2. $\operatorname{nil}_{\pi} H_{n}(K(A, m)) \leq \sum_{j=0}^{n} \operatorname{nil}_{\pi} E_{n-j, j}^{2} \quad(m \geq 1)$. (We use the notation in the proof of Proposition 1.)

Proof. It is known that the spectral sequence invoked in the previous proposition consists of $\pi$-modules (the actions are induced by $w$ ) $E_{r, s}^{k}$ and the differentials $d_{r, s}^{k}$ are $\pi$-module homomorphisms. Also,

$$
E_{r+2, s-1}^{2} \stackrel{d_{r+2, s-1}^{2}}{\longrightarrow} E_{r, s}^{2} \stackrel{d_{r, s}^{2}}{\longrightarrow} E_{r-2, s+1}^{2}
$$

and

$$
E_{r, s}^{3}=\frac{\operatorname{ker}\left(d_{r, s}^{2}\right)}{\operatorname{im}\left(d_{r+2, s-1}^{2}\right)} .
$$

Thus we get the exact sequence of $\pi$-modules,

$$
0 \rightarrow \operatorname{im} d_{r+2, s_{1}}^{2} \rightarrow \operatorname{ker} d_{r, s}^{2} \rightarrow E_{r, s}^{3} \rightarrow 0 .
$$


It follows from Proposition I.4.3 [HMR] that

$$
\operatorname{nil}_{\pi} E_{r, s}^{3} \leq \operatorname{nil}_{\pi} \operatorname{ker} d_{r, s}^{2} \leq \operatorname{nil}_{\pi} E_{r, s}^{2} .
$$

It is now readily seen, by induction, that

$$
\operatorname{nil}_{\pi} E_{r, s}^{k} \leq \operatorname{nil}_{\pi} E_{r, s}^{2} \quad \forall k \geq 2 .
$$

In particular, $\operatorname{nil}_{\pi} E_{r, s}^{\infty} \leq \operatorname{nil}_{\pi} E_{r, s}^{2}$. Finally, recalling the filtration

$$
E_{0, n}^{\infty} \subset F_{1, n-1} \subset \cdots \subset F_{n, 0}=H_{n}(K(A, m)),
$$

the exact sequence of $\pi$-modules

$$
F_{i-1, n-i+1} \rightarrow F_{i, n-i} \rightarrow E_{i, n-i}^{\infty},
$$

and using several times Proposition I.4.3 [HMR], we obtain

$$
\operatorname{nil}_{\pi} H_{n}(K(A, m)) \leq \sum_{j=0}^{m} \operatorname{nil}_{\pi} E_{n-j, j}^{\infty} \leq \sum_{j=0}^{n} \operatorname{nil}_{\pi} E_{n-j, j}^{2} .
$$

Theorem 3. If $\operatorname{nil}_{\pi} A=$ nil $w=c \geq 2$ and $m \geq 2$, then

(i) $\operatorname{nil}_{\pi} H_{n}(K(A, m)) \leq c$ if $0 \leq n<2 m$.

(ii) nil $_{\pi} H_{n}(K(A, m)) \leq c(c+1) / 2$ if either $n=2 m$ or $n=2 m+1$.

(iii) $\operatorname{nil}_{\pi} H_{2 m+2}(K(A, m)) \leq c^{2}$ if $m \geq 3$.

(iv) $\operatorname{nil}_{\pi} H_{6}(K(A, 2)) \leq c(c+1)(c+2) / 6$.

(v) $\operatorname{nil}_{\pi} H_{2 m+3}(K(A, m)) \leq 2 c^{2}-c$ if $m \geq 4$.

(vi) $\operatorname{nil}_{\pi} H_{9}(K(A, 3)) \leq c / 6\left(c^{2}=9 c-4\right)$.

(vii) $\operatorname{nil}_{\pi} H_{7}(K(A, 2)) \leq c / 3\left(c^{2}+3 c-1\right)$.

Proof. In the Serre spectral sequence we have

$$
E_{n-j, j}^{2}=H_{n-j}\left(K(A / \Gamma, m) ; H_{j}(K(\Gamma, m))\right) ;
$$

so $E_{n, 0}^{2} \cong H_{n}(K(A / \Gamma, m))$ and $E_{0, n}^{2} \cong H_{n}(K(\Gamma, m)$ ) (isomorphisms of $\pi$ modules). Thus,

(i) The inequality is trivially true if $0 \leq n<m$, and if $n=m$ the result follows from the Hurewicz isomorphism since $w_{n}=w$. In fact, $\operatorname{nil}_{\pi} H_{m}(K(A, m))$ $=c \leq c$. It remains to consider the case in which $m<n<2 m$. Fix $j$, such that $0<j<n$.

If $0<j<m$ then $H_{j}(K(\Gamma, m))=(0)$, so $E_{n-j, j}^{2}=(0)$.

If $m \leq j<n$ then $0<n-j<m$. Therefore $H_{n-j}(K(A / \Gamma, m))=0$, so $E_{n-j, j}^{2}=0$.

It follows from Lemma 2 that

$$
\operatorname{nil}_{\pi} H_{n}(K(A, m)) \leq \operatorname{nil}_{\pi} E_{0, n}^{2}+\operatorname{nil}_{\pi} E_{n, 0}^{2} \leq 1+\operatorname{nil}_{\pi} H_{n}(K(A / \Gamma, m))
$$

since $\pi$ acts trivially on $\Gamma$. It turns out, then, by induction on $c$ that $\operatorname{nil}_{\pi} H_{n}(K(A, m)) \leq c$.

(ii) Again, $0<j<m \Rightarrow E_{n-j, j}^{2}=0$ and $m<j<n=2 m \Rightarrow 0<n-j<m$. Therefore $E_{n-j, j}^{2}=0$ and

$$
E_{m, m}^{2} \cong H_{m}(K(A / \Gamma, m)) \otimes H_{m}(K(\Gamma, m)) \cong A / \Gamma \otimes \Gamma .
$$


We now invoke the inequality (1.3) from [HRS] in order to state the nil $E_{m, m}^{2}$ $\leq \operatorname{nil}_{\pi} A / \Gamma=c-1$. Thus, by using Lemma 2, we obtain

$$
\operatorname{nil}_{\pi} H_{2 m}(K(A, m)) \leq 1+(c-1)+\operatorname{nil}_{\pi} H_{2 m}(K(A / \Gamma, m)) .
$$

It follows, therefore, by induction, that

$$
\operatorname{nil}_{\pi} H_{2 m}(K(A, m)) \leq 1+(c-1)+\frac{(c-1) c}{2}=\frac{c(c+1)}{2} .
$$

For $n=2 m+1$, we have again $E_{n-j, j}^{2}=0$, if either $0<j<m$ or $m+1<$ $j<2 m+1$. Also $E_{m, m+1}^{2}=0$, for $H_{m+1}(K(\Gamma, m))=0$ (Hurewicz) $(m \geq 2)$ and

$$
E_{m+1, m}^{2} \cong \operatorname{Tor}\left(H_{m}(K(A / \Gamma, m)) ; H_{m}(\Gamma, m)\right) \cong \operatorname{Tor}(A / \Gamma, \Gamma) \text {. }
$$

As it can be checked, ${ }^{1}$

$$
\operatorname{nil}_{\pi} \operatorname{Tor}(A, B) \leq \operatorname{nil}_{\pi} A+\operatorname{nil}_{\pi} B-1,
$$

whence

$$
\operatorname{nil}_{\pi} E_{m+1, m}^{2} \leq \operatorname{nil}_{\pi} A / \Gamma=c-1 .
$$

Hence,

$$
\operatorname{nil}_{\pi} H_{2 m+1}(K(A, m)) \leq 1+(c-1)+\operatorname{nil}_{\pi} H_{2 m+1}(K(A / \Gamma, m)),
$$

whence, by induction,

$$
\operatorname{nil}_{\pi} H_{2 m+1}(K(A, m)) \leq 1+(c-1)+\frac{(c-1) c}{2}=\frac{c(c+1)}{2} .
$$

(iii) Now let us suppose that $m \geq 3$ and $n=2 m+2$.

$$
\begin{aligned}
& E_{n-j, j}^{2}=0 \text { if either } 0<j<m \text { or } m+2<j<2 m+2 . \\
& E_{m+1, m+1}^{2}=0\left(\text { for } H_{m+1}(K(\Gamma, m))=0\right) . \\
& E_{m+2, m}^{2} \cong H_{m+2}(K(A / \Gamma, m)) \otimes H_{m}(K(\Gamma, m)) .
\end{aligned}
$$

Therefore, $E_{m+2, m}^{2} \cong H_{m+2}(K(A / \Gamma, m)) \otimes \Gamma$. Taking into account that $m \geq 3$ (therefore, $m+2<2 m$ ) it follows from (i) that

$$
\left.\mathrm{nil}_{\pi} E_{m+2, m}^{2} \leq \operatorname{nil}_{\pi} H_{m+2}(K(A / \Gamma, m)) \leq c-1 \text { for } \operatorname{nil}_{\pi} A / \Gamma=c-1\right) .
$$

Also, $E_{m, m+2}^{2} \cong A / \Gamma \otimes H_{m+2}(K(\Gamma, m))$. So, $\operatorname{nil}_{\pi} E_{m, m+2}^{2} \leq \operatorname{nil}_{\pi} A / \Gamma=c-1$. Thus,

$$
\begin{aligned}
\operatorname{nil}_{\pi} H_{2 m+2}(K(A, m)) & \leq 1+(c-1)+(c-1)+\operatorname{nil}_{\pi} H_{2 m+2}(K(A / \Gamma, m)), \\
& =(2 c-1)+\operatorname{nil}_{\pi} H_{2 m+2}(K(A / \Gamma, m)) .
\end{aligned}
$$

Then, by induction,

$$
\operatorname{nil}_{\pi} H_{2 m+2}(K(A, m)) \leq(2 c-1)+(c-1)^{2}=c^{2} .
$$

(iv) $m=2$ and $n=2 m+2=6$. The same kind of calculation that we have used in (iii) leads us to conclude that $\mathrm{nil}_{\pi} E_{4,2}^{2} \leq \operatorname{nil}_{\pi} H_{4}(K(A / \Gamma, 2)) \leq$ $c(c-1) / 2$ (due to (ii)) and $\mathrm{nil}_{\pi} E_{2,4}^{2} \leq c-1$. So,

$$
\begin{aligned}
\operatorname{nil}_{\pi} H_{6}(K(A, 2)) & \leq 1+(c-1)+\frac{(c-1) c}{2}+\operatorname{nil}_{\pi} H_{6}(K(A / \Gamma, 2)) \\
& =\frac{c(c+1)}{2}+\operatorname{nil}_{\pi} H_{6}(K(A / \Gamma, 2)) .
\end{aligned}
$$

${ }^{1}$ I would like to thank the referee for pointing out this improvement of my earlier inequality. 
Thus,

$$
\operatorname{nil}_{\pi} H_{6}(K(A, 2)) \leq \frac{c(c+1)}{2}+\frac{(c-1) c(c+1)}{6}=\frac{c(c+1)(c+2)}{6} .
$$

(v) $m \geq 4$ and $n=2 m+3$.

$E_{2 m+3-j, j}^{2}=0$ if either $0<j<m$ or $m+3<j<2 m+3$ or $j=m+1$. $E_{m, m+3}^{2} \cong A / \Gamma \otimes H_{m+3}(K(\Gamma, m))$.

$E_{m+1, m+2}^{2} \cong \operatorname{Tor}\left(A / \Gamma, H_{m+2}(K(\Gamma, m))\right.$, and the following sequence is exact:

$$
H_{m+3}(K(A / \Gamma, m)) \otimes \Gamma \rightarrow E_{m+3, m}^{2} \rightarrow \operatorname{Tor}\left(H_{m+2}(K(A / \Gamma, m)), \Gamma\right) .
$$

So, due to Lemma 2 ,

$$
\begin{aligned}
\operatorname{nil}_{\pi} H_{2 m+3}(K(A, m)) \leq & 1+\operatorname{nil}_{\pi} H_{2 m+3}(K(A / \Gamma, m))+\operatorname{nil}_{\pi} A / \Gamma \\
& +\operatorname{nil}_{\pi} A / \Gamma+\operatorname{nil}_{\pi} H_{m+3}(K(A / \Gamma, m)) \\
& +\operatorname{nil}_{\pi} H_{m+2}(K(A / \Gamma, m)) \cdots
\end{aligned}
$$

By (i) and remembering that $m \geq 4$,

$$
\operatorname{nil}_{\pi} H_{2 m+3}(K(A, m)) \leq(4 c-3)+\operatorname{nil}_{\pi} H_{2 m+3}(K(A / \Gamma, m)) .
$$

Then,

$$
\operatorname{nil}_{\pi} H_{2 m+3}(K(A, m)) \leq(4 c-3)+\left\{2(c-1)^{2}-(c-1)\right\}=c(2 c-1) .
$$

(vi) $m=3$ and $n=2 m+3=9$.

Using $(*)$ from previous item we get

$$
\begin{aligned}
\operatorname{nil}_{\pi} H_{9}(K(A, 3)) \leq & \operatorname{nil}_{\pi} H_{9}(K(A / \Gamma, 3))+(2 c-1) \\
& +\operatorname{nil}_{\pi} H_{6}(K(A / \Gamma, 3))+\operatorname{nil}_{\pi} H_{5}(K(A / \Gamma, 3)) \\
\leq & \operatorname{nil}_{\pi} H_{9}(K(A / \Gamma, 3))+(2 c-1)+\frac{c(c-1)}{2}+c-1,
\end{aligned}
$$

due to items (i) and (ii) as well. Therefore,

$$
\operatorname{nil}_{\pi} H_{9}(K(A, 3)) \leq \frac{c^{2}+5 c-4}{2}+\operatorname{nil}_{\pi} H_{9}(K(A / \Gamma, 3))
$$

whence

$$
\operatorname{nil}_{\pi} H_{9}(K(A, 3)) \leq \frac{c^{2}+5 c-4}{2}+\frac{c-1}{6}\left[(c-1)^{2}+9(c-1)-4\right]=\frac{c}{6}\left(c^{2}+9 c-4\right) \text {. }
$$

(vii) $m=2$ and $n=2 m+3=7$. Again, we invoke (*) from item (v), as well as item (ii) to get

$$
\begin{aligned}
\operatorname{nil}_{\pi} H_{7}(K(A, 2)) \leq & (2 c-1)+\operatorname{nil}_{\pi} H_{7}(K(A / \Gamma, 2)) \\
& +\operatorname{nil}_{\pi} H_{5}(K(A / \Gamma, 2))+\operatorname{nil}_{\pi} H_{4}(K(A / \Gamma, 2)) \\
\leq & (2 c-1)+\operatorname{nil}_{\pi} H_{7}(K(A / \Gamma, 2))+\frac{c(c-1)}{2}+\frac{c(c-1)}{2} \\
= & \left(c^{2}+c-1\right)+\operatorname{nil}_{\pi} H_{7}(K(A / \Gamma, 2)),
\end{aligned}
$$

whence

$$
\begin{aligned}
\operatorname{nil}_{\pi} H_{7}(K(A, 2)) & \leq\left(c^{2}+c-1\right)+\frac{(c-1)}{3}\left\{(c-1)^{2}+3(c-1)-1\right\} \\
& =\frac{c}{3}\left(c^{2}+3 c-1\right) .
\end{aligned}
$$


Example 4. Let $\mathbf{Z} \stackrel{w}{\longrightarrow} \operatorname{Aut}(\mathbf{Z} \oplus \mathbf{Z})$ be the action given by

$$
w(1) \cdot(1,0)=(1,1) \text { and } w(1) \cdot(0,1)=(0,1) \text {, }
$$

that is,

$$
M=\left[\begin{array}{ll}
1 & 0 \\
1 & 1
\end{array}\right]
$$

is the matrix attached to the automorphism $w(1)$.

$$
\left(M-I_{2}\right)^{2}=0 \text { and } M-I_{2} \neq 0 \Rightarrow w \text { is nilpotent and nil } w=2 .
$$

Let $X=K(\mathbf{Z} \oplus \mathbf{Z}, 2) \cong K(\mathbf{Z}, 2) \otimes K(\mathbf{Z}, 2)$ and set $w_{n}: \mathbf{Z} \rightarrow \operatorname{Aut}\left(H_{n}(X)\right)$ the action induced by $w$. In order to calculate nil $w_{n}$, we remember that $H_{*}(K(\mathbf{Z}, 2))$ is the divided polynomial algebra with generators of all even degrees (i.e., $\left.x_{2 i} \cdot x_{2 j}=\left(\left(\begin{array}{c}i+j \\ i\end{array}\right)\right) x_{2(i+j)}\right)$.

It follows from the definition that $w_{n}$ is compatible with the multiplicative structure in $H_{n}(X)$.

Moreover, $w_{2}=w$ (Hurewicz) and remembering that

$$
\begin{aligned}
& H_{2}(X) \cong {\left[H_{0}(K(\mathbf{Z}, 2)) \otimes H_{2}(K(\mathbf{Z}, 2))\right] } \\
& \oplus\left[H_{2}(K(\mathbf{Z}, 2)) H_{0}(K(\mathbf{Z}, 2))\right] \quad \text { (Künneth) }
\end{aligned}
$$

we get

$$
w_{2}(1) \cdot\left(x_{2} \otimes 1\right)=x_{2} \otimes 1, \quad w_{2}(1)\left(1 \otimes x_{2}\right)=1 \otimes x_{2}+x_{2} \otimes 1 .
$$

It is known that $\left\{1 \otimes x_{4}, x_{2} \otimes x_{2}, x_{4} \otimes 1\right\}$ is a base in $H_{4}(X)$ (where $x_{4}$ is a generator in $\left.H_{4}(K(\mathbf{Z}, 2))\right)$.

Now,

$$
\begin{aligned}
2 w_{4}(1)\left(1 \otimes x_{4}\right) & =w_{4}(1)\left(1 \otimes 2 x_{4}\right)=w_{4}(1)\left[\left(1 \otimes x_{2}\right) \cdot\left(1 \otimes x_{2}\right)\right] \\
& =w_{2}(1)\left(1 \otimes x_{2}\right) \cdot w_{2}(1)\left(1 \otimes x_{2}\right) \\
& =\left[1 \otimes x_{2}+x_{2} \otimes 1\right] \cdot\left[1 \otimes x_{2}+x_{2} \otimes 1\right] \\
& =1 \otimes 2 x_{4}+2 x_{2} \otimes x_{2}+2 x_{4} \otimes 1 .
\end{aligned}
$$

So, $w_{4}(1)\left(1 \otimes x_{4}\right)=1 \otimes x_{4}+x_{2} \otimes x_{2}+x_{4} \otimes 1$.

A similar calculation shows us that $w_{4}(1)\left(x_{2} \otimes x_{2}\right)=x_{2} \otimes x_{2}+2 x_{4} \otimes 1$ and $w_{4}(1)\left(x_{4} \otimes 1\right)=x_{4} \otimes 1$.

Therefore,

$$
M_{4}=\left[\begin{array}{lll}
1 & 0 & 0 \\
1 & 1 & 0 \\
1 & 2 & 1
\end{array}\right]
$$

is the matrix attached to $w_{4}(1)$ relative to the base previously indicated.

Thus $\left(M_{4}-I_{3}\right)^{3}=0$ and $\left(M_{4}-I_{3}\right)^{2} \neq 0$, whence nil $w_{4}=3=2(2+1) / 2$.

Likewise, fixing the base $\left\{1 \otimes x_{6}, x_{2} \otimes x_{4}, x_{4} \otimes x_{2}, x_{6} \otimes 1\right\}$ in $H_{6}(X)$, we get

$$
M_{6}=\left[\begin{array}{llll}
1 & 0 & 0 & 0 \\
1 & 1 & 0 & 0 \\
1 & 2 & 1 & 0 \\
1 & 3 & 3 & 1
\end{array}\right]
$$

following the same steps of the previous argument. 
Then, $\left(M_{6}-I_{4}\right)^{4}=0$ and $\left(M_{6}-I_{4}\right)^{3} \neq 0$, whence nil $w_{6}=4=$ $\sum_{j=1}^{2} j(j+1) / 2$.

Actually, this example can be generalized.

Example 5. Let $\mathbf{Z} \stackrel{w}{\longrightarrow} \operatorname{Aut}\left(\mathbf{Z}^{c}\right)$ be the action defined by the matrix

$$
M=\left[\begin{array}{ccccc}
1 & 0 & 0 & \cdots & 0 \\
1 & 1 & 0 & \cdots & 0 \\
1 & 1 & 1 & \cdots & 0 \\
\vdots & \vdots & \vdots & & \vdots \\
1 & 1 & 1 & \cdots & 1
\end{array}\right]
$$

Thus nil $w=c$, since $\left(M-I_{c}\right)^{c}=0$ and $\left(M-I_{c}\right)^{c-1} \neq 0$.

Let $X_{c}=K\left(\mathbf{Z}^{c}, 2\right) \cong K(\mathbf{Z}, 2)^{c}$ and take $w_{n}: \mathbf{Z} \rightarrow \operatorname{Aut}\left(H_{n}\left(X_{c}\right)\right)$ to be the action induced by $w$.

Calculations similar to the ones used in the previous example show us that

$$
\left.\operatorname{nil}_{\mathbf{Z}} H_{4}\left(X_{c}\right)=\operatorname{nil} w_{4}=\frac{c(c+1)}{2}=\text { rank of } H_{4}\left(X_{c}\right) \quad \text { (by induction on } c\right) \text {, }
$$

and

$$
\operatorname{nil}_{\mathbf{Z}} H_{6}\left(X_{c}\right)=\text { nil } w_{6}=\sum_{j=1}^{c} \frac{j(j+1)}{2}=\text { rank of } H_{6}\left(X_{c}\right) \text {. }
$$

(Notice that by the Künneth Formula, we obtain rank

$$
\begin{aligned}
H_{6}\left(X_{c}\right) & =\sum_{i=0}^{3} \operatorname{rank} H_{2 i}\left(X_{c-1}\right) \\
& \left.=1+(c-1)+\frac{c(c-1)}{2}+\sum_{j=1}^{c-1} \frac{j(j+1)}{2} \quad \text { (induction) }=\sum_{j=1}^{c} \frac{j(j+1)}{2} .\right)
\end{aligned}
$$

$M_{4}(c)$ and $M_{6}(c)$ turn out to be triangular, by induction on $c$.

Remark. This example shows us that the inequalities obtained in Theorem 3(ii) $(n=4$ and $m=2)$ and in Theorem 3(iv) are the best ones.

Proposition 6. If nil $w=\operatorname{nil}_{\pi} A=c \geq 2$ then

(i) $\operatorname{nil}_{\pi} H_{2}(A) \leq c(c+1) / 2$ and

(ii) $\operatorname{nil}_{\pi} H_{3}(A) \leq \sum_{j=1}^{c} j^{2}=c(c+1)(2 c+1) / 6$.

Proof. We invoke the Lyndon-Hochschild-Serre spectral sequence attached to the exact sequence $\Gamma \rightarrow A \rightarrow A / \Gamma$ (where $\Gamma=\Gamma_{w}^{c} \neq 0$ ) to get $E_{r, s}^{2}=$ $H_{r}\left(A / \Gamma ; H_{s}(\Gamma)\right)$ with trivial coefficients.

(i) $E_{2,0}^{2}=H_{2}(A / \Gamma) ; E_{0,2}^{2} \cong H_{2}(\Gamma) ; E_{1,1}^{2} \cong A / \Gamma \otimes \Gamma$.

So, $\operatorname{nil}_{\pi} H_{2}(A) \leq 1+(c-1)+\operatorname{nil}_{\pi} H_{2}(A / \Gamma)$ due to Lemma 2 and the inequality (1.3) [HRS].

Thus, we get (by induction again)

$$
\operatorname{nil}_{\pi} H_{2}(A) \leq c+\frac{(c-1) c}{2}=\frac{c(c+1)}{2} .
$$


(ii) $E_{3,0}^{2} \cong H_{3}(A / \Gamma) ; E_{0,3}^{2} \cong H_{3}(\Gamma) ; E_{1,2}^{2} \cong A / \Gamma \otimes H_{2}(\Gamma)$ and $H_{2}(A / \Gamma) \otimes$ $\Gamma \rightarrow E_{2,1}^{2} \rightarrow \operatorname{Tor}(A / \Gamma, \Gamma)$ is exact.

So,

$$
\operatorname{nil}_{\pi} H_{3}(A) \leq 1+\operatorname{nil}_{\pi} H_{3}(A / \Gamma)+(c-1)+(c-1)+\operatorname{nil}_{\pi} H_{2}(A / \Gamma) .
$$

Now nil $H_{2}(A / \Gamma) \leq(c-1) c / 2$ due to the previous case, and $c \geq 2 \Leftrightarrow c / 2 \leq$ $c-1 \Leftrightarrow(c-1) c / 2 \leq(c-1)^{2}$.

So $\operatorname{nil}_{\pi} H_{2}(A / \Gamma) \leq(c-1)^{2}$. It follows that

$$
\operatorname{nil}_{\pi} H_{3}(A) \leq(2 c-1)+(c-1)^{2}+\operatorname{nil}_{\pi} H_{3}(A / \Gamma)=c^{2}+\operatorname{nil}_{\pi} H_{3}(A / \Gamma) .
$$

Therefore,

$$
\operatorname{nil}_{\pi} H_{3}(A) \leq c^{2}+\frac{(c-1) c(2 c-1)}{6}=\frac{c(c+1)(2 c+1)}{6} .
$$

From now on $X$ will denote a connected CW-complex, $\widetilde{X}$ its universal covering; $\pi=\pi_{1}(X)$ and $\pi_{n}=\pi_{n}(X)$.

We are also going to use the Postnikov decomposition of $\widetilde{X}$ and its dual Cartan-Serre-Whitehead decomposition. They will be indicated by

(Postnikov)

$$
K\left(\pi_{m}, m\right) \hookrightarrow \begin{gathered}
\vdots \\
\tilde{X}_{m} \\
\downarrow \\
\tilde{X}_{m-1} \\
\downarrow \\
\vdots \\
\\
\\
\\
\\
\tilde{\tilde{X}}_{2}=K(\pi, 2) \\
\downarrow \\
*
\end{gathered}
$$

(Dual)

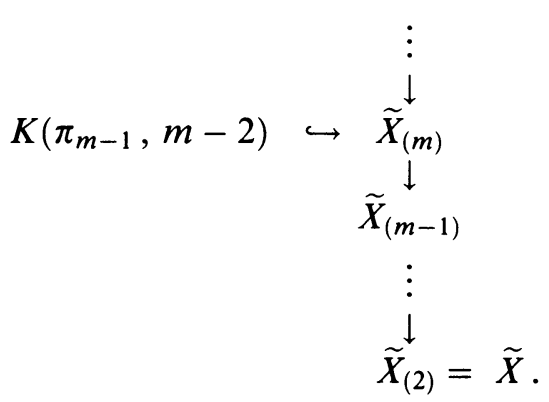

Here we should recall that Lemma II.2.18 and Remark 2.19 from [HMR] show us that $\pi$ acts nilpotently on $\pi_{n} \forall n, 2 \leq n \leq k \Leftrightarrow \pi$ acts nilpotently on $H_{n}(\tilde{X}) \quad \forall n, 2 \leq n \leq k$.

Our goal now is to obtain some results comparing such nilpotency classes.

Thus, we suppose from now on that $\pi$ acts nilpotently on $\pi_{n} \forall n \geq 2$. Concerning the previous decompositions, we prove 2 lemmas to be used later. 
Lemma 7. (i) $\operatorname{nil}_{\pi} H_{m+1}\left(\widetilde{X}_{m}\right) \leq \operatorname{nil}_{\pi} H_{m+1}\left(\widetilde{X}_{m+1}\right)$.

$$
\begin{aligned}
\operatorname{nil}_{\pi} H_{m+2}\left(\widetilde{X}_{m}\right) \leq & \operatorname{nil}_{\pi} H_{m+2}\left(\widetilde{X}_{m-1}\right)+\operatorname{nil}_{\pi} H_{m+2}\left(K\left(\pi_{m}(X), m\right)\right) \\
& +\operatorname{nil}_{\pi}\left[\pi_{2}(X) \otimes \pi_{m}(X)\right]
\end{aligned}
$$

$$
\begin{aligned}
\operatorname{nil}_{\pi} H_{m+3}\left(\tilde{X}_{m}\right) \leq & \operatorname{nil}_{\pi} H_{m+3}\left(\tilde{X}_{m-1}\right)+\operatorname{nil}_{\pi} H_{m+3}\left(K\left(\pi_{m}(X), m\right)\right) \\
& +\operatorname{nil}_{\pi}\left[H_{3}\left(\tilde{X}_{m-1}\right) \otimes \pi_{m}(X)\right]+\operatorname{nil}_{\pi} \operatorname{Tor}\left(\pi_{2}(X), \pi_{m}(X)\right)
\end{aligned}
$$

(iv) $\operatorname{nil}_{\pi} H_{m+4}\left(\widetilde{X}_{m}\right) \leq \operatorname{nil}_{\pi} H_{m+4}\left(\widetilde{X}_{m-1}+\operatorname{nil}_{\pi} H_{m+4}\left(K\left(\pi_{m}(X), m\right)\right.\right.$

$$
\begin{aligned}
& +\operatorname{nil}_{\pi} \pi_{2}(X) \otimes H_{m+2}\left(K\left(\pi_{m}(X), m\right)\right) \\
& +\operatorname{nil}_{\pi} H_{4}\left(\tilde{X}_{m-1}\right) \otimes \pi_{m}(X) \\
& +\operatorname{nil}_{\pi} \operatorname{Tor}\left(H_{3}\left(\tilde{X}_{m-1}\right), \pi_{m}(X)\right), \forall m \geq 3 .
\end{aligned}
$$

Proof. It is enough to take the Serre spectral sequence attached to the fibration

$$
\begin{aligned}
& K\left(\pi_{m}(X), m\right) \hookrightarrow \tilde{X}_{m} \\
& \tilde{X}_{m-1}^{\downarrow} \text {. }
\end{aligned}
$$

The argument is similar to the one used in the previous Proposition 6.

\section{Corollary 8.}

(i) $\operatorname{nil}_{\pi} H_{5}\left(\tilde{X}_{3}\right) \leq \operatorname{nil}_{\pi} H_{5}\left(K\left(\pi_{2}, 2\right)\right)+\operatorname{nil}_{\pi} H_{5}\left(K\left(\pi_{3}, 3\right)\right)+\operatorname{nil}_{\pi} \pi_{2} \otimes \pi_{3}$.

(ii) $\operatorname{nil}_{\pi} H_{6}\left(\widetilde{X}_{3}\right) \leq \operatorname{nil}_{\pi} H_{6}\left(K\left(\pi_{2}, 2\right)\right)+\operatorname{nil}_{\pi} H_{6}\left(K\left(\pi_{3}, 3\right)\right)+\operatorname{nil}_{\pi} \operatorname{Tor}\left(\pi_{2}, \pi_{3}\right)$.

$$
\begin{aligned}
\operatorname{nil}_{\pi} H_{7}\left(\tilde{X}_{3}\right) \leq & \operatorname{nil}_{\pi} H_{7}\left(K\left(\pi_{2}, 2\right)\right)+\operatorname{nil}_{\pi} H_{7}\left(K\left(\pi_{3}, 3\right)\right) \\
& +\operatorname{nil}_{\pi} \pi_{2} \otimes H_{5}\left(K\left(\pi_{3}, 3\right)\right)+\operatorname{nil}_{\pi} H_{4}\left(K\left(\pi_{2}, 2\right)\right) \otimes \pi_{3} .
\end{aligned}
$$

$$
\begin{aligned}
\operatorname{nil}_{\pi} H_{6}\left(\widetilde{X}_{4}\right) \leq & \operatorname{nil}_{\pi} H_{6}\left(K\left(\pi_{4}, 4\right)\right)+\operatorname{nil}_{\pi} H_{6}\left(K\left(\pi_{3}, 3\right)\right) \\
& +\operatorname{nil}_{\pi} H_{6}\left(K\left(\pi_{2}, 2\right)\right)+\operatorname{nil}_{\pi} \pi_{2} \otimes \pi_{4}+\operatorname{nil}_{\pi} \operatorname{Tor}\left(\pi_{2}, \pi_{3}\right)
\end{aligned}
$$

$$
\begin{aligned}
\operatorname{nil}_{\pi} H_{7}\left(\widetilde{X}_{4}\right) \leq & \operatorname{nil}_{\pi} H_{7}\left(K\left(\pi_{2}, 2\right)\right)+\operatorname{nil}_{\pi} H_{7}\left(K\left(\pi_{3}, 3\right)\right) \\
& +\operatorname{nil}_{\pi} H_{7}\left(K\left(\pi_{4}, 4\right)\right)+\operatorname{nil}_{\pi} H_{3}(X) \otimes \pi_{4} \\
& +\operatorname{nil}_{\pi} \operatorname{Tor}\left(\pi_{2}, \pi_{4}\right)+\operatorname{nil}_{\pi} \pi_{2} \otimes H_{5}\left(K\left(\pi_{3}, 3\right)\right) \\
& +\operatorname{nil}_{\pi} H_{4}\left(K\left(\pi_{2}, 2\right)\right) \otimes \pi_{3} .
\end{aligned}
$$

(vi)

$$
\begin{aligned}
\operatorname{nil}_{\pi} H_{7}\left(\tilde{X}_{5}\right) \leq & \operatorname{nil}_{\pi} H_{7}\left(K\left(\pi_{2}, 2\right)+\operatorname{nil}_{\pi} H_{7}\left(K\left(\pi_{3}, 3\right)\right)\right. \\
& +\operatorname{nil}_{\pi} H_{7}\left(K\left(\pi_{4}, 4\right)\right)+\operatorname{nil}_{\pi} H_{7}\left(K\left(\pi_{5}, 5\right)\right)+\operatorname{nil}_{\pi} \pi_{2} \otimes \pi_{5} \\
& +\operatorname{nil}_{\pi} \pi_{4} \otimes H_{3}(\tilde{X})+\operatorname{nil}_{\pi} \operatorname{Tor}\left(\pi_{2}, \pi_{4}\right)+\operatorname{nil}_{\pi} \pi_{2} \otimes H_{5}\left(K\left(\pi_{3}, 3\right)\right) \\
& +\operatorname{nil}_{\pi} \pi_{3} \otimes H_{4}\left(K\left(\pi_{2}, 2\right)\right) .
\end{aligned}
$$




\section{Lemma 9.}

$$
\begin{aligned}
\operatorname{nil}_{\pi} H_{m+1}\left(\tilde{X}_{(m)}\right) \leq & \operatorname{nil}_{\pi} H_{m+1}\left(\tilde{X}_{(m-1)}\right)+\operatorname{nil}_{\pi} H_{m+1}\left(K\left(\pi_{m-1}, m-2\right)\right) \\
& +\operatorname{nil}_{\pi} H_{3}\left(\widetilde{X}_{(m-1)}\right) \otimes \pi_{m-1} \\
& +\operatorname{nil}_{\pi} \operatorname{Tor}\left(H_{2}\left(\widetilde{X}_{(m-1)}\right), \pi_{m-1}\right), \forall m \geq 3
\end{aligned}
$$

$$
\begin{aligned}
\operatorname{nil}_{\pi} H_{m+2}\left(\widetilde{X}_{(m)}\right) \leq & \operatorname{nil}_{\pi} H_{m+2}\left(\tilde{X}_{(m-1)}\right)+\operatorname{nil}_{\pi} H_{m+2}\left(K\left(\pi_{m-1}, m-2\right)\right) \\
& +\operatorname{nil}_{\pi} H_{2}\left(\widetilde{X}_{(m-1)}\right) \otimes H_{m}\left(K\left(\pi_{m-1}, m-2\right)\right) \\
& +\operatorname{nil}_{\pi} H_{4}\left(\widetilde{X}_{(m-1)}\right) \otimes \pi_{m-1} \\
& +\operatorname{nil}_{\pi} \operatorname{Tor}\left(H_{3}\left(\tilde{X}_{(m-1)}\right), \pi_{m-1}\right), \quad \forall m \geq 3
\end{aligned}
$$

Proof. Just take the fibration

$$
K\left(\pi_{m-1}(X), m-2\right) \hookrightarrow \begin{gathered}
\tilde{X}_{(m)} \\
\downarrow \\
\tilde{X}_{(m-1)} .
\end{gathered}
$$

\section{Corollary 10.}

$$
\begin{aligned}
\operatorname{nil}_{\pi} H_{4}\left(\tilde{X}_{(3)}\right) \leq & \operatorname{nil}_{\pi} H_{4}(\tilde{X})+\operatorname{nil}_{\pi} H_{4}\left(K\left(\pi_{2}, 1\right)\right) \\
& +\operatorname{nil}_{\pi} H_{3}(\tilde{X}) \otimes \pi_{2}+\operatorname{nil}_{\pi} \operatorname{Tor}\left(H_{2}(\tilde{X}) ; \pi_{2}\right) .
\end{aligned}
$$

$$
\begin{aligned}
\operatorname{nil}_{\pi} H_{5}\left(\widetilde{X}_{(3)}\right) \leq & \operatorname{nil}_{\pi} H_{5}(\tilde{X})+\operatorname{nil}_{\pi} H_{5}\left(K\left(\pi_{2}, 1\right)\right)+\operatorname{nil}_{\pi} H_{2}(\tilde{X}) \otimes H_{3}\left(\pi_{2}, 1\right) \\
& +\operatorname{nil}_{\pi} H_{4}(\tilde{X}) \otimes \pi_{2}+\operatorname{nil}_{\pi} \operatorname{Tor}\left(H_{3}(\tilde{X}), \pi_{2}\right)
\end{aligned}
$$

(iii) $\quad \operatorname{nil}_{\pi} H_{5}\left(\widetilde{X}_{(4)}\right) \leq \operatorname{nil}_{\pi} H_{5}\left(\widetilde{X}_{(3)}\right)+\operatorname{nil}_{\pi} H_{5}\left(K\left(\pi_{3}, 2\right)\right)+\operatorname{nil}_{\pi} \pi_{3} \otimes \pi_{3}$.

(iv) $\quad \operatorname{nil}_{\pi} H_{m+1}\left(\widetilde{X}_{(m)}\right) \leq \operatorname{nil}_{\pi} H_{m+1}\left(\widetilde{X}_{(m-1)}\right)+\operatorname{nil}_{\pi} H_{m+1}\left(K\left(\pi_{m-1}, m-\right.\right.$ 2), provided that $m \geq 5$.

$$
\begin{aligned}
\operatorname{nil}_{\pi} H_{6}\left(\widetilde{X}_{(4)}\right) \leq & \operatorname{nil}_{\pi} H_{6}\left(\tilde{X}_{(3)}\right)+\operatorname{nil}_{\pi} H_{6}\left(K\left(\pi_{3}, 2\right)\right) \\
& +\operatorname{nil}_{\pi} H_{4}\left(\widetilde{X}_{(3)}\right) \otimes \pi_{3}+\operatorname{nil}_{\pi} \operatorname{Tor}\left(\pi_{3}, \pi_{3}\right) .
\end{aligned}
$$

$$
\operatorname{nil}_{\pi} H_{7}\left(\widetilde{X}_{(5)}\right) \leq \operatorname{nil}_{\pi} H_{7}\left(\widetilde{X}_{(4)}\right)+\operatorname{nil}_{\pi} H_{7}\left(K\left(\pi_{4}, 3\right)\right)+\operatorname{nil}_{\pi} \pi_{4} \otimes \pi_{4} .
$$

$$
\operatorname{nil}_{\pi} H_{m+2}\left(\widetilde{X}_{(m)}\right) \leq \operatorname{nil}_{\pi} H_{m+2}\left(\tilde{X}_{(m-1)}\right)+\operatorname{nil}_{\pi} H_{m+2}\left(K \left(\pi_{m-1}, m-\right.\right.
$$
2)).

Lemma 11. (i) $\operatorname{nil}_{\pi} H_{m}(\widetilde{X}) \leq \operatorname{nil}_{\pi} \pi_{m}(X)+\operatorname{nil}_{\pi} H_{m}\left(\tilde{X}_{m-1}\right), \quad m \geq 3$.

$$
\begin{aligned}
\operatorname{nil}_{\pi} \pi_{m} \leq & \operatorname{nil}_{\pi} H_{m}\left(\widetilde{X}_{(m-1)}\right)+\operatorname{nil}_{\pi} H_{m}\left(K\left(\pi_{m-1}, m-2\right)\right) \\
& +\operatorname{nil}_{\pi} H_{2}\left(\tilde{X}_{(m-1)}\right) \otimes \pi_{m-1}, \quad \forall m \geq 3 .
\end{aligned}
$$


Proof. For (i) take the fibration

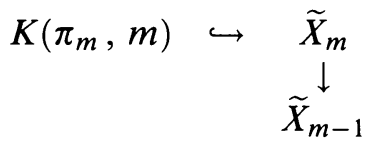

and for (ii) use

$$
K\left(\pi_{m-1}, m-2\right) \hookrightarrow \begin{gathered}
\tilde{X}_{(m)} \\
\downarrow \\
\tilde{X}_{(m-1)} .
\end{gathered}
$$

Theorem 12. Under the conditions that $\pi$ acts nilpotently on $\pi_{n}, 2 \leq n \leq 7$, we have

$$
\begin{aligned}
& \operatorname{nil}_{\pi} H_{3}(\tilde{X}) \leq \operatorname{nil}_{\pi} \pi_{3}(X) \leq \operatorname{nil}_{\pi} H_{3}(\tilde{X})+\operatorname{nil}_{\pi} H_{2}(\tilde{X}) \otimes H_{2}(\tilde{X}) \\
&+\operatorname{nil}_{\pi} H_{3}\left(H_{2}(\tilde{X})\right) \\
& \operatorname{nil}_{\pi} \pi_{4} \leq \operatorname{nil}_{\pi} H_{4}(\tilde{X})+\operatorname{nil}_{\pi} H_{4}\left(K\left(\pi_{3}, 2\right)\right)+\operatorname{nil}_{\pi} H_{4}\left(K\left(\pi_{2}, 1\right)\right) \\
&+ \operatorname{nil}_{\pi} H_{3}(\tilde{X}) \otimes H_{2}(\tilde{X})+\operatorname{nil}_{\pi} \operatorname{Tor}\left(H_{2}(\tilde{X}), H_{2}(\tilde{X})\right) \\
& \operatorname{nil}_{\pi} H_{4}(\tilde{X}) \leq \operatorname{nil}_{\pi} \pi_{4}(X)+\operatorname{nil}_{\pi} H_{4}\left(K\left(\pi_{2}, 2\right)\right) \\
& \\
& \operatorname{nil}_{\pi} H_{5}(\tilde{X}) \leq \operatorname{nil}_{\pi} \pi_{5}(X)+\operatorname{nil}_{\pi} \pi_{2} \otimes \pi_{3}+\operatorname{nil}_{\pi} H_{5}\left(K\left(\pi_{2}, 2\right)\right) \\
&+\operatorname{nil}_{\pi} H_{5}\left(K\left(\pi_{3}, 3\right)\right) \\
& \operatorname{nil}_{\pi} H_{6}(\tilde{X}) \leq \operatorname{nil}_{\pi} \pi_{6}(X)+\operatorname{nil}_{\pi} \pi_{2} \otimes \pi_{4}+\operatorname{nil}_{\pi} H_{6}\left(K\left(\pi_{2}, 2\right)\right) \\
&+\operatorname{nil}_{\pi} H_{6}\left(K\left(\pi_{3}, 3\right)\right)+\operatorname{nil}_{\pi} H_{6}\left(K\left(\pi_{4}, 4\right)\right) \\
& \operatorname{nil}_{\pi} \pi_{7}(X)+\operatorname{nil}_{\pi} \pi_{2} \otimes \pi_{5}+\operatorname{nil}_{\pi} \pi_{4} \otimes H_{3}(\tilde{X}) \\
&\left.+\operatorname{nil}_{\pi} \operatorname{Tor}_{2}, \pi_{4}\right)+\operatorname{nil}_{\pi} \pi_{3} \otimes H_{4}\left(K\left(\pi_{2}, 2\right)\right) \\
&+\operatorname{nil}_{\pi} \pi_{2} \otimes H_{5}\left(K\left(\pi_{3}, 3\right)\right)+\operatorname{nil}_{\pi} H_{7}\left(K\left(\pi_{2}, 2\right)\right) \\
&+\operatorname{nil}_{\pi} H_{7}\left(K\left(\pi_{3}, 3\right)\right)+\operatorname{nil}_{\pi} H_{7}\left(K\left(\pi_{4}, 4\right)\right) \\
&+\operatorname{nil}_{\pi} H_{7}\left(K\left(\pi_{5}, 5\right)\right) .
\end{aligned}
$$

Proof. To get (i) we recall that $\tilde{X}_{(2)}=\tilde{X}$ and that $H_{3}\left(\tilde{X}_{2}\right)=H_{3}\left(K\left(\pi_{2}, 2\right)\right)=0$. Then, it is enough to use Lemma 11(i), (ii).

(ii) results from the use of Lemma 11 (ii) and Corollary 10(i).

(iii) follows from Lemma 11(i) and Lemma 7(i).

(iv) follows from Lemma 11(i) together with Lemma 7(i) and Corollary 8(i).

(v) is a consequence of Lemma 11(i), Lemma 7(i), and Corollary 8(iv).

Finally, (vi) results from the use of Lemma 11(i) together with Lemma 7(i) and Corollary $8(\mathrm{vi})$.

Remark. The inequalities obtained for $\operatorname{nil}_{\pi} \pi_{n}$ and $\operatorname{nil}_{\pi} H_{m}(\tilde{X})$ turn out to be very complicated if $n>4$ and $m>7$. As a matter of fact, if $n=4$ and $m=7$, they are not so simple, as the items (ii) and (vi) from the previous theorem show us. 
Example 13. Let $X$ be a connected $\mathrm{CW}$-complex such that $\pi_{1}(X) \cong \mathbf{Z}, \pi_{2}(X) \cong \mathbf{Z}^{c}, \pi_{i}(X)=0, i>2$, and the action of $\pi_{1}(X)$ or $\pi_{2}(X)$ is given by $M$ as in Example 5 .

Thus, $\widetilde{X}=K\left(\mathbf{Z}^{c}, 2\right)$ and according to Example 5 we have $\operatorname{nil}_{\pi_{1}(X)} \pi_{2}(X)=c$ and $\operatorname{nil}_{\pi_{1}(X)} H_{4}(\tilde{X})=c(c+1) / 2$.

Owing to the fact that $\pi_{4}(X)=0$, we may state that the inequality (iii) obtained in Theorem 12 is in this case an equality.

Likewise, this example yields a situation in which inequality $(v)$ is an equality!

\section{REFERENCES}

[H] Peter J. Hilton, On G-spaces, Bol. Soc. Brasil Mat. 7 (1976), 65-73.

[HMR] P. Hilton, G. Mislin, and J. Roitberg, Localization of nilpotent groups and spaces, Notas Mat., vol. 15, North Holland, Amsterdam, 1975.

[HRS] P. Hilton, J. Roitberg, and D. Singer, On G-spaces, Serre classes, and G-nilpotency, Math. Proc. Cambridge Philos. Soc. 84 (1978), 443-454.

[W] George W. Whitehead, Elements of homotopy theory, Graduate Texts in Math., SpringerVerlag, New York, Heidelberg, and Berlin, 61 (1978).

Instituto de Matemática e Estatística, Universidade de São Paulo, Caixa Postal 20570, CEP 01498, SÃo Paulo, Brasil 